\title{
Structural stability of Cutibacterium acnes acyl carrier protein studied using CD and NMR spectroscopy
}

\author{
Ahjin Jang ${ }^{1}$, Dasom Cheon ${ }^{1}$, Eunha Hwang ${ }^{2}$ and Yangmee Kim ${ }^{1 *}$ (1)
}

\begin{abstract}
To survive in diverse environments, bacteria adapt by changing the composition of their cell membrane fatty acids. Compared with aerobic bacteria, Cutibacterium acnes has much greater contents of branched-chain fatty acids (BCFAs) in the cell membrane, which helps it survive in anaerobic environments. To synthesize BCFAs, C. acnes acyl carrier protein (CaACP) has to transfer growing branched acyl intermediates from its hydrophobic cavity to fatty acid synthases. CaACP contains an unconserved, distinctive Cys50 in its hydrophobic pocket, which corresponds to Leu in other bacterial acyl carrier proteins (ACPs). Herein, we investigated the substrate specificity of CaACP and the importance of Cys50 in its structural stability. We mutated Cys50 to Leu (C50L mutant) and measured the melting temperatures (Tms) of both CaACP and the C50L mutant by performing circular dichroism experiments. The Tm of CaACP was very low $\left(49.6^{\circ} \mathrm{C}\right)$, whereas that of $\mathrm{C} 50 \mathrm{~L}$ mutant was $55.5^{\circ} \mathrm{C}$. Hydrogen/deuterium exchange experiments revealed that wild-type CaACP showed extremely fast exchange rates within 50 min, whereas amide peaks of the C50L mutant in the heteronuclear single quantum coherence spectrum remained up to $200 \mathrm{~min}$, thereby implying that Cys50 is the key residue contributing to the structural stability of CaACP. We also monitored chemical shift perturbations upon apo to holo, apo to butyryl, and apo to isobutyryl conversion, confirming that CaACP can accommodate isobutyryl BCFAs. These results provide a preliminary understanding into the substrate specificity of CaACPs for the production of BCFAs necessary to maintain cell membrane fluidity under anaerobic environments.
\end{abstract}

Keywords: Fatty acid synthesis, Cutibacterium acnes, Acyl carrier protein, Substrate specificity, NMR spectroscopy

\section{Introduction}

Cutibacterium acnes, previously known as Propionibacterium acnes, is an important member of the human skin microbiota. C. acnes thrives in anaerobic conditions and produces various metabolites (Aubin et al. 2017). It plays important roles in the production of vitamin $\mathrm{B}_{12}$ as well as probiotics used commonly in the food and cosmetic industries. C. acnes is an opportunistic pathogen causing various skin conditions depending on the growth environment (dry/oily sebum) (Boisrenoult 2018). Fatty acids

\footnotetext{
*Correspondence: ymkim@konkuk.ac.kr

${ }^{1}$ Department of Bioscience and Biotechnology, Konkuk University,

Seoul 05029, Republic of Korea

Full list of author information is available at the end of the article
}

present in sebaceous niches are important energy sources for colonizing bacteria; furthermore, bacteria secrete lipases that allow for the uptake of these fatty acids. Antibiotics, including tetracycline, clindamycin, and rifampicin, are generally used to treat $C$. acnes infection. However, the emergence of antibiotic-resistant $C$. acnes has accelerated the development of effective antibiotics targeting $C$. acnes. It has been recently reported that $C$. acnes is involved in other inflammatory disorders of the digestive system as well as in cardiovascular and orthopedic disorders (Aubin et al. 2017; Boisrenoult 2018; Gharamti and Kanafani 2017). Therefore, to develop antibiotics against $C$. acnes infection, it is important to gain a deeper understanding into mechanism of how fatty acid synthesis (FAS) occurs in the pathogen. 
Membrane lipid homeostasis is crucial for the survival of bacteria. By adjusting their membrane composition, bacteria can adapt to environmental changes (Zhang and Rock 2008). Bacteria are easily exposed to toxic organic compounds under anaerobic environments. A series of different organic compounds has been reported to be approximately three times more fatal to anaerobic bacteria than to aerobic bacteria. Anaerobic bacteria degrade various organic solvents and compounds. The membrane fluidity of $C$. acnes supports cell viability and the degradation of toxic compounds (Duldhardt et al. 2010; Kaspar 1982). Moreover, C. acnes has a high content of branched-chain fatty acids (BCFAs) in its membrane, which provides fluidity for adaptation to anaerobic growth environments. Straight chain saturated fatty acids (SFAs) are stacked tightly, making the bilayer more stable and rigid; when cis-unsaturated fatty acids (UFAs) or BCFAs add to the chain, the order of the bilayer is interrupted, which increases the permeability of membrane and lowers the transition temperature. Compared with iso BCFAs, anteiso BCFAs yield more flexibility to membranes; this is attributed to the methyl branch present at the end of anteiso BCFAs (Zhang and Rock 2008). In whole cells of various Cutibacteria strains, $\mathrm{C}_{15}$ BCFA is the most abundant fatty acid ( 50\%); 12-methyltetradecanoic acid $\left(\mathrm{a}-\mathrm{C}_{15}\right)$ and 13-methyltetradecanoic acid $\left(\mathrm{i}-\mathrm{C}_{15}\right)$ are present in different proportions (Moss et al. 1969).

Acyl carrier protein (ACP) is an important cofactor in FAS and delivers an acyl group to other enzymes or intermediates (Ploskon et al. 2010). $\beta$-ketoacyl ACP synthase III (KASIII, FabH) starts the initiation steps by binding two carbon units to malonyl-ACP, thus beginning the elongation cycle of FAS. In most bacteria, KASIII exhibits substrate specificity for the elongation step by adjusting the conformation of several loops. For example, palmitic, hexadecenoic, and $\beta$-hydroxymyristic SFAs have a $50.4 \%$, $12.6 \%$, and $12.0 \%$ predominance in the fatty acid composition of Escherichia coli, respectively (Shaw and Ingraham 1965). E. coli KASIII (EcKASIII) has a relatively lower preference for butyryl CoA than for acetyl CoA as a substrate (Heath and Rock 1996). EcKASIII is important in the condensation step of FAS, which involves the transfer of two carbons from acyl-CoA to malonyl-ACP (Lai and Cronan 2003). EcKASI and EcKASII also perform further elongation steps after reduction and dehydration (Rock and Cronan 1996); this is because, unlike EcKASIII, EcKASI and EcKASII prefer alkyl groups of alkyl-ACP to those of alkyl-CoAs. EcKASIII also accepts propionyl CoA and butyryl CoA as substrates; however, the condensation step with butyryl CoA is very inefficient as compared to that with acetyl CoA and propionyl CoA (Heath and Rock 1996).
Previously, we also determined the crystal structure of CaKASIII and reported that CaKASIII prefers isobutyryl CoA as its substrate (Cheon et al. 2019). The cavity of CaKASIII is wider than that of Mycobacterium tuberculosis KASIII (MtKASIII) and EcKASIII; this is because CaKASIII has a Ser97 located at the end of the cavity, instead of the Thr97 in MtKASIII and Phe87 in EcKASIII (Scarsdale et al. 2001; Qiu et al. 1999). A superimposed model of CaKASIII with EcKASIII and MtKASIII has a large space in the active site, which is enough to accommodate BCFAs, such as isobutyryl and isovaleryl moieties, as well as butyryl chains.

FAS is relatively unstudied in $C$. acnes compared to that in other bacteria. To obtain insights into the substrate specificity of $C$. acnes for producing BCFAs under anaerobic environments, we analyzed the amino acid sequence of CaACP as compared to the sequences of other ACPs and found that $\mathrm{CaACP}$ has an unconserved, distinctive Cys50. We investigated the importance of Cys50 in the structural stability of CaACP using circular dichroism (CD) and hydrogen/deuterium (H/D) exchange experiments. We also investigated the substrate specificity of CaACP using chemical shift perturbation (CSP). Our study confirmed that $\mathrm{CaACP}$ has a substrate specificity for BCFAs and offered essential insights into the development of antibiotics against $C$. acnes at the molecular level.

\section{Results}

\section{Comparison with other ACP sequences}

Because the amino acid sequence of a protein is closely related to its functions, we first compared the amino acid sequence of CaACP with that of the ACPs of various other bacteria (Fig. 1). CaACP showed high sequence similarity with other bacterial ACPs (Fig. 1), sharing the $4^{\prime}$-phosphopantetheine prosthetic (4'-PP) group attached to the conserved serine residue (Ser40 in CaACP) at the beginning of the second helix near the conserved AspSer-Leu (DSL) motif marked in the green box. Distinctively, only CaACP has a unique unconserved Cys50 in helix II, marked in the red box in Fig. 1. Most bacterial ACPs have Leu at the same position. The role of Leu42 and Leu46 in the hydrophobic cavity of E. coli has been investigated extensively using molecular dynamics simulation (Chan et al. 2008). EcACP has two subpockets that use the same entrance. Subpocket I is surrounded by helix II, III, and IV, whereas subpocket II by helix I, II, and IV. When the acyl chain enters subpocket I, both Leu42 and Leu46 switch the dihedral angle to open the pathway for the acyl chains. Leu42 and Leu46 play important roles as gating residues in the opening of two subpockets that can be occupied by the acyl chains by changing the orientation of Leu side chains (Chan et al. 2008). 


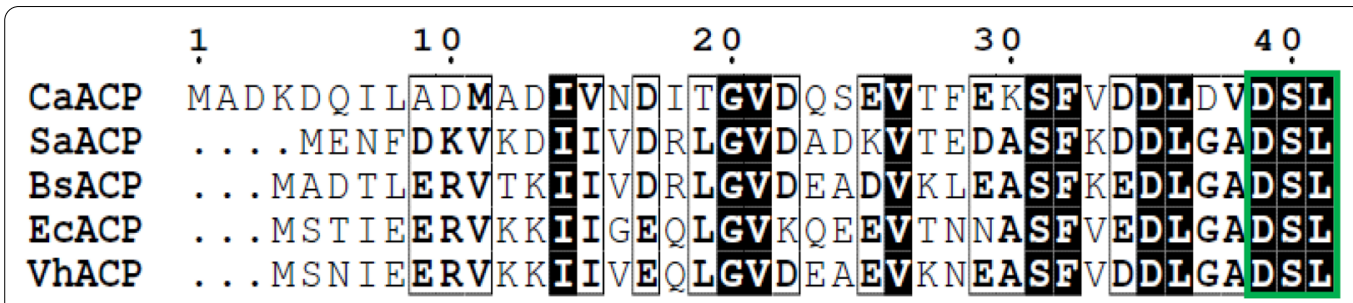

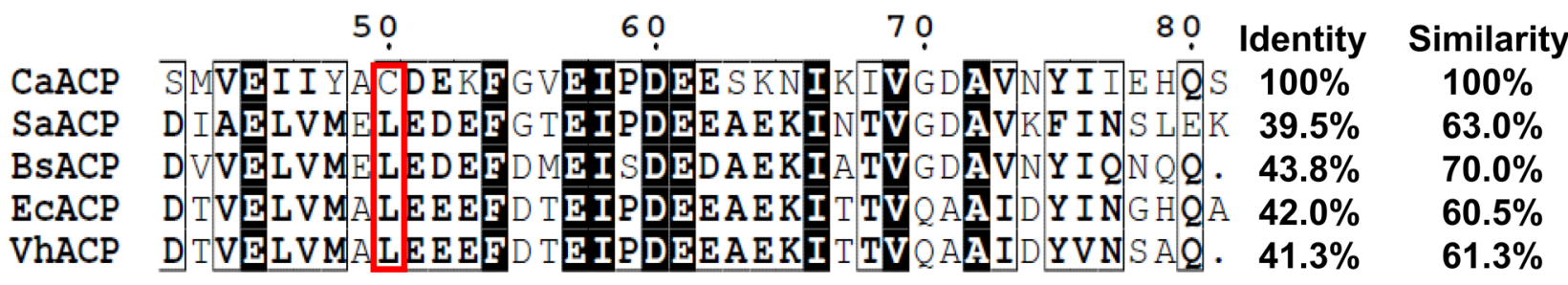

Fig. 1 Comparison of bacterial ACP sequences. The black boxes show the 100\% conserved residues. Residues having similar properties are shown in white boxes. Based on the CaACP, the identity and similarity of each ACP is marked. The conserved DSL motif is marked in a green box. The unique Cys50 residue in CaACP is marked in red

Proper folding of ACPs requires the hydrophobic interactions in the hydrophobic cavity to include growing acyl chains formed by amino acids such as Leu, Ile, and Val, which are aliphatic branched-chain amino acids. The relative volumes of Leu, Ile, and Val are $63.6 \%, 63.6 \%$, and $47.7 \%$, respectively, compared to that of $\operatorname{Trp}(100 \%)$ (Bogardt et al. 1980). Cys is an amphipathic amino acid and has a thiol side chain; it occupies lesser space than Leu or Phe does, resulting in reduced hydrophobic interactions in the cavity. Therefore, CaACP might be less stable than the other bacterial ACPs.

\section{Thermal stability of CaACP}

To investigate the thermal stability and role of the unique Cys50 in CaACP, we investigated the secondary structure and Tm of CaACP. We mutated Cys50 to Leu (C50L mutant) to obtain a sequence same as that of EcACP, which is conserved in other ACPs (Fig. 1). We performed $\mathrm{CD}$ experiments for $\mathrm{CaACP}$ and the C50L mutant. The double minima at $205 \mathrm{~nm}$ and $222 \mathrm{~nm}$ are shown for both proteins from 190 to $260 \mathrm{~nm}$, confirming the characteristics of an $\alpha$-helix structure (Fig. 2a). The CaACP C50L mutant showed more characteristics of $\alpha$-helix compared to the wild type. To investigate the thermostability (a)

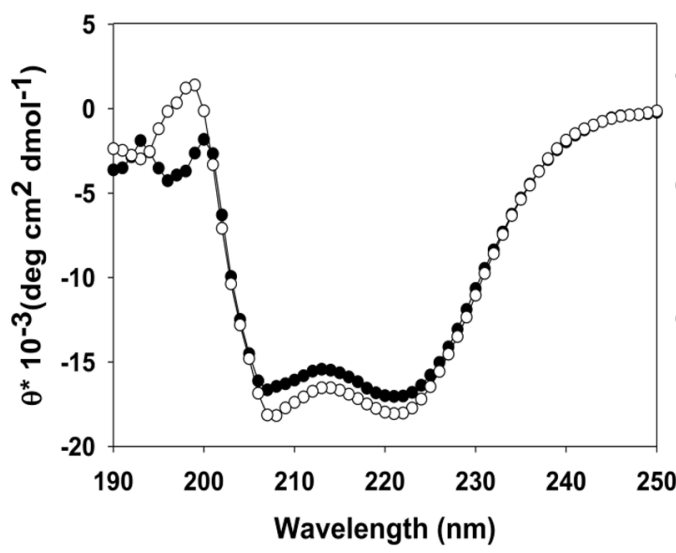

(b)

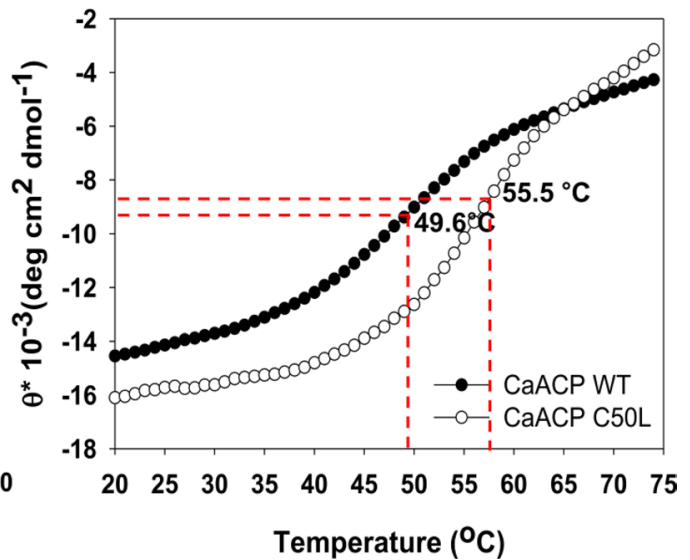

Fig. 2 The secondary structure and melting temperature of CaACP. a CD spectra of the CaACP WT and C50L mutant from 190 to $260 \mathrm{~nm}$ wavelength. $\mathbf{b}$ The melting curves of the WT and C50L mutant. All samples were in $25 \mathrm{mM} \mathrm{Mes}$ and $5 \mathrm{mM} \mathrm{CaCl}_{2}$ buffer (pH 6.1) 
of CaACP in comparison to that of $\mathrm{C} 50 \mathrm{~L}$, we measured their Tms using CD; we monitored the mean residue ellipticity at $222 \mathrm{~nm}$, which reflects temperature-induced folding changes of proteins. The $\mathrm{Tm}$ of $\mathrm{CaACP}$ was $49.6{ }^{\circ} \mathrm{C}$, whereas that of $\mathrm{C} 50 \mathrm{~L}$ was $55.5^{\circ} \mathrm{C}$, implying that Cys50 is the main factor underlying the low thermal stability of CaACP. Leu, Ile, and Val are typical hydrophobic aliphatic amino acids, whereas Cys is a hydrophilic amino acid. Therefore, the presence of Cys50 in the hydrophobic cavity may loosen the hydrophobic packing, thereby affecting substrate specificity.

\section{H/D exchange experiments}

To investigate the structural stability of CaACP in further detail using NMR spectroscopy, we completed the backbone assignment of CaACP spectra using HNCO, $\mathrm{HNCACB}$, and $\mathrm{CBCA}(\mathrm{CO}) \mathrm{NH}$ experiments. In addition, the assignment of $\mathrm{H}_{\alpha}$ was completed using $2 \mathrm{D}^{1} \mathrm{H}-{ }^{1} \mathrm{H}$ nuclear Overhauser effect spectroscopy and total correlation spectroscopy experiments. Using chemical shifts of ${ }^{13} \mathrm{C}_{\alpha},{ }^{13} \mathrm{C}_{\beta}$, and ${ }^{1} \mathrm{H}_{\alpha}$, chemical shift indexes (CSIs) were calculated (Wishart and Sykes 1994; Wishart et al. 1992) and used to predict the secondary structure of CaACP with algorithm of the Protein Energetic Conformational Analysis from NMR chemical shifts (PECAN). PECAN predicts the most favorable secondary structure in terms of energy based on amino acid sequence and statistical energy function of each residues (Eghbalnia et al. 2005). $\mathrm{CaACP}$ was observed to contain four helical regions: helix I (Lys4-Thr19), helix II (Ser40-Phe54), helix III (Asp60-Asn65), and helix IV (Gly70-His79) (Fig. 3). This suggests that the overall folding of $\mathrm{CaACP}$ is very similar to that of other bacterial ACPs having four helix bundles. Therefore, we confirmed that Cys50 is located in the

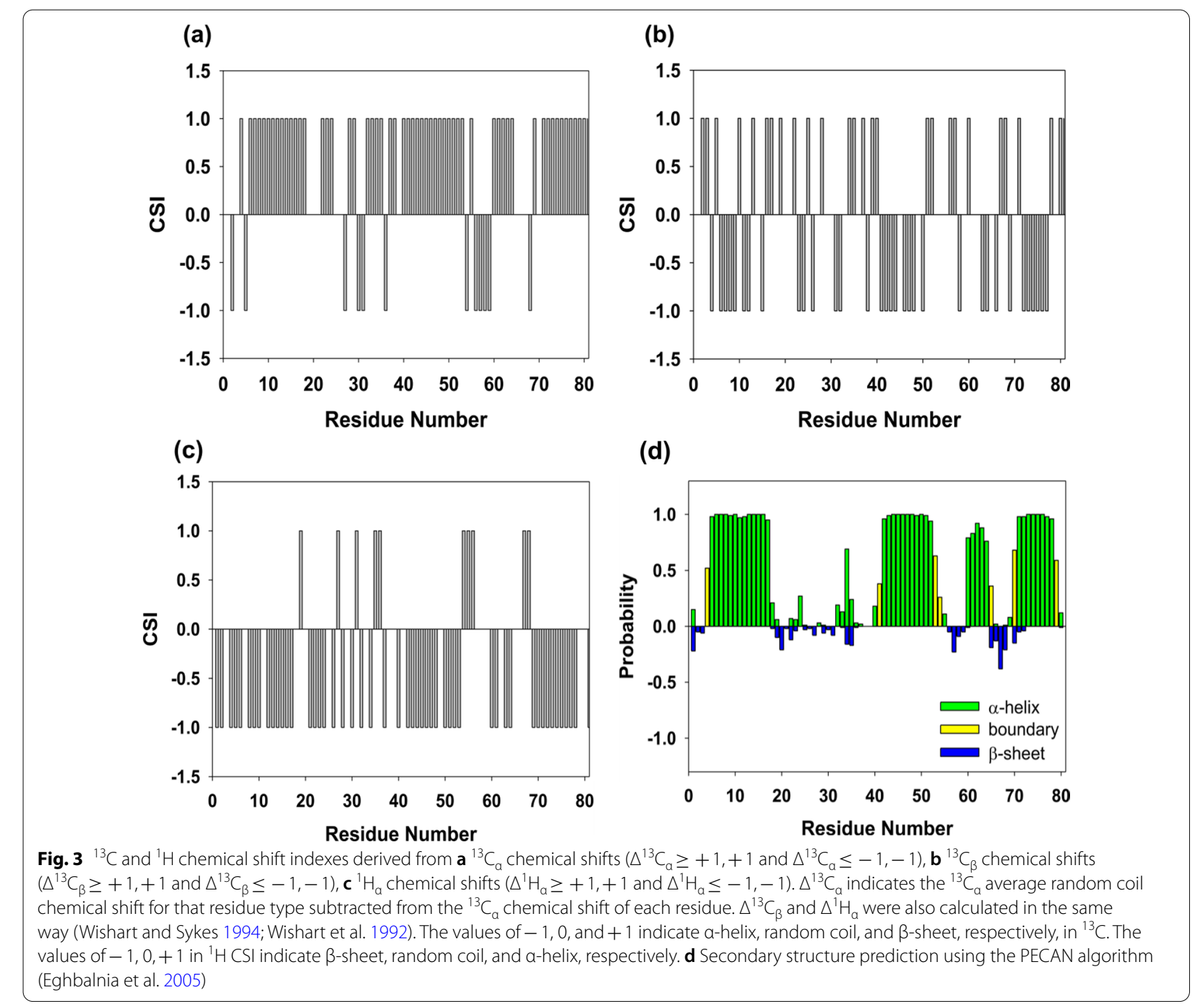


middle of helix II and plays important roles in the stability of the hydrophobic cavity of CaACP.

Because the Tm of CaACP C50L was $6{ }^{\circ} \mathrm{C}$ higher than that of CaACP WT, we conducted H/D exchange experiments to understand the role of Cys50 in the structure of CaACP. Figure 4a shows the heteronuclear single quantum coherence (HSQC) spectrum of CaACP with a marking for Ser40, which is the 4'-PP group attachment site, and Cys50, which is crucial in the thermal stability of CaACP. WT CaACP showed an extremely fast H/D exchange rate. In the WT CaACP, amide peaks of only 10 residues--Ile7, Ile14, Val15, Val44, Gly45, Ile47, Ala49, Val73, Ile76, and Ile77--remained after $10 \mathrm{~min}$; however, the peaks disappeared within $50 \mathrm{~min}$. In contrast, the C50L mutant showed a relatively slower H/D exchange rate. As shown in Fig. 4b, c, only Ile47 and Ala49 in helix II of CaACP WT remained after 30 min, whereas more than 10 peaks from residues such as Ile7, Ile14, Val44, Leu50, Val69, and Ile74 in helix I, II, and IV forming a hydrophobic cavity remained after $30 \mathrm{~min}$. However, in the C50L mutant, only three residues--Ile14 in helix I, Ala49 and Leu50 in helix II--remained after $120 \mathrm{~min}$ (Fig. 4d). These results imply that the mutated C50L can stabilize a protein by ensuring tight packing in the hydrophobic cavity of $\mathrm{CaACP}$.

\section{Substrate specificity of CaACP as studied by CSPs}

Because ACP is an essential cofactor that delivers acyl groups to various fatty acid synthases, the motional property of ACP is very important as a carrier. $\mathrm{CaACP}$ is converted to an active holo-form via the attachment of a 4'-PP group to the conserved Ser40, resulting in CSPs due to the hydrophobic interactions between the $4^{\prime}$-PP group and the residues in the hydrophobic cavity. Upon conversion from apo- to holo-CaACP, a large CSP was observed mainly for the residues near the Ser40-binding site and near the entrance of the cavity (Fig. 5a, d).

To clarify the substrate specificity of CaACPs with linear or branched acyl chains, the CSPs in ${ }^{1} \mathrm{H}-{ }^{15} \mathrm{~N}$ HSQC spectra upon conversion from apo to butyryl and isobutyryl forms of CaACP were investigated. The entrance of acyl chains into the hydrophobic cavity of ACP formed by four helices resulted in CSPs, as compared to that in the apo form. Figure $5 \mathrm{~d}-\mathrm{f}$ provide a structural representation of the CSPs in CaACP on the homology modeled structure. When a linear butyryl chain is attached to Ser40, the residues in helix I and helix III as well as in helix II showed large CSPs, implying that CaACP can accommodate a butyryl group deep in the hydrophobic cavity (Fig. 5b, e). Similar to the FAS in most bacteria, CaACP carries linear fatty acyl chains to fatty acid synthases.

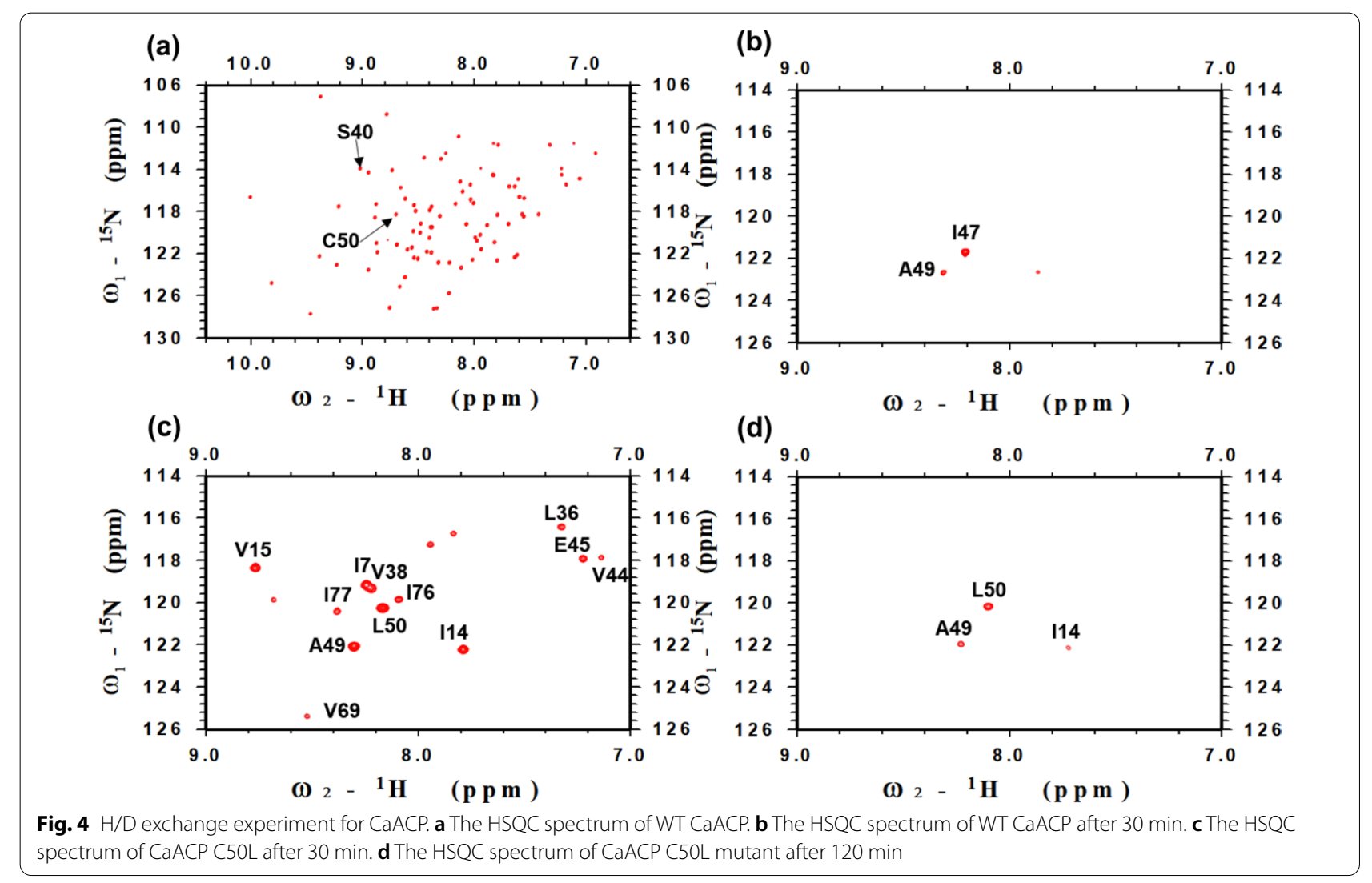




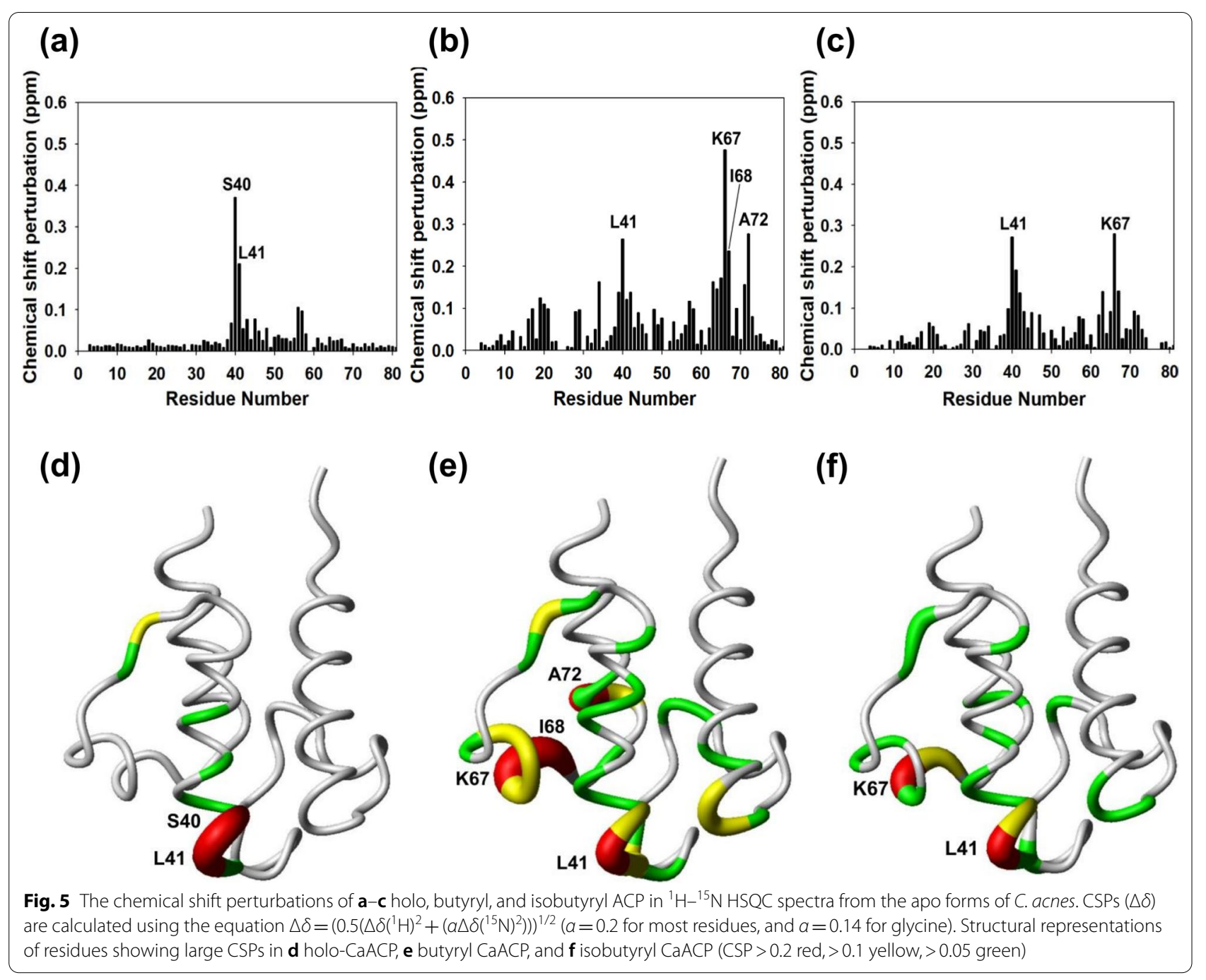

However, as shown in Fig. 5c, f, CaACP also showed large CSPs upon conversion from apo to isobutyryl forms in a pattern similar to that of the butyryl form, thereby confirming the substrate specificity of CaACP for BCFAs. Because the buried side chains of the hydrophobic residues form a hydrophobic cavity surrounded by four amphipathic helixes and loops (Roujeinikova et al. 2007), all the residues showing large CSPs were hydrophobic residues forming hydrophobic interactions with hydrophobic acyl chains. Furthermore, Val56 and Glu57 at loop II between helix II and helix III also showed CSPs upon conversion to the holo, butyryl, and isobutyryl forms, implying that flexibility of loop II is important in acyl binding.

\section{Discussion}

Because C. acnes thrives in anaerobic environments, high membrane fluidity is necessary to extract excessive metabolites and organic solvents that interrupt cell viability (Duldhardt et al. 2010; Kaspar 1982). The high ratio of BFCAs, especially a-C15 and i-C15, which comprise $50 \%$ of the $C$. acnes membrane, provide high fluidity to the bacterial membrane. In our previous study, we confirmed the substrate specificity of CaKASIII for BCFAs. The crystal structure of CaKASIII showed that the unconserved Ser97, which is positioned at the bottom part of the active site cavity, contributes to yield a spherelike wider cavity compared to that in other bacterial KAS III. Mycobacteria have a thick membrane wall comprising long acyl chains such as mycolic acids. Unusually, mycobacteria have both a type I and a type II fatty-acid synthase. MtKASIII activity connects between the type I and type II FAS. Some unique structural characteristics of MtKASIII preferentially utilize long chain acyl-CoA substrates like myristoyl-CoA. Binding channel 1 is very similar to EcKASIII but, binding channel 2 has distinctive feature for the unique substrate specificities of MtKASIII. In case of EcKASIII, Phe87 in this binding site obstructs 
binding of straight fatty acid chains longer than 4 carbons. In case of MtKASIII, Thr87 which is smaller can allow binding of longer chain fatty acids and make space for two more carbons than myristoyl group (Scarsdale et al. 2001). In case of CaKASIII, there is Ser97 located at the bottom of the cavity of CaKASIII, which allows to accept short branched-chain CoAs rather than straight, longer acyl-CoAs (Cheon et al. 2019).

CaACP is an important cofactor in C. acnes FAS; it transfers BFCA intermediates to fatty acid synthases. Although the sequence of CaACP is highly similar to that of other bacterial ACPs and the overall folding of $\mathrm{CaACP}$ is very similar to that of other bacterial ACPs, the structure and amino acid sequence of CaACP may play important roles in its substrate specificity. Interestingly, only CaACP possesses unique Cys50 residues at helix II, which corresponds to Leu in other bacterial ACPs. Previous molecular dynamics simulation studies on acylated EcACP reported that there are two subpockets in the hydrophobic cavity of EcACP and that the gate opening of these pockets is controlled by Leu 42 and Leu 46 in EcACP (Chan et al. 2008). Therefore, the unique Cys50 in $\mathrm{CaACP}$ may play critical roles in the structure of $\mathrm{CaACP}$; thus, we investigated its role in the structure and substrate specificity of CaACP.

We predicted the thermal stability through circular dichroism $(\mathrm{CD})$ by gradually raising the temperature from 20 to $75{ }^{\circ} \mathrm{C}$ to monitor thermal denaturation of structure (Niklasson et al. 2015). On the other hand, we utilized hydrogen/deuterium (H/D) exchange experiments to investigate the protein folding at $25{ }^{\circ} \mathrm{C}$. As protein is dissolved in $\mathrm{D}_{2} \mathrm{O}$, labile hydrogen atoms on the protein will exchange with deuterium, exchange rates of each backbone amide protons provide information on the local backbone conformation and dynamics. Therefore, we tried to monitor the H/D exchange rates of amide protons which play roles in structural stability of CaACP (Krishna et al. 2004). CD experiments on WT $\mathrm{CaACP}$ and $\mathrm{C50L}$ mutants revealed that the C50L mutation results in the increase of thermal stability. Compared to the Tm of other bacterial ACPs [EcACP $\left(67.2^{\circ} \mathrm{C}\right)$, Staphylococcus aureus ACP (SaACP, $\left.67.6{ }^{\circ} \mathrm{C}\right)$, Acinetobacter baumannii ACP (AbACP, $\left.68.0{ }^{\circ} \mathrm{C}\right)$, Enterococcus faecalis ACP (EfACP, $78.8^{\circ} \mathrm{C}$ ), and Thermotoga maritima ACP (TmACP, $\left.101.4{ }^{\circ} \mathrm{C}\right)$ ], CaACP shows a much lower thermal stability, implying that Cys50 may loosen the hydrophobic cavity (Park et al. 2016; Choi et al. 2021; Lee et al. 2020). In addition, the H/D exchange rate of CaACP WT was very fast, and the resonances of all residues disappeared within $50 \mathrm{~min}$. In case of thermophilic ACPs such as EfACP and TmACP, the amide protons inside of the hydrophobic cavity were hyperprotected and remained stable for more than a week, implying that those ACPs underwent stable protein folding (Park et al. 2016; Lee et al. 2020). In case of mesophilic ACPs such as EcACP, the residues at helix I, helix II, and helix IV forming the hydrophobic cavity had a high protection factor, thereby revealing stable protein structure (Andrec et al. 1995). In the case of Vibrio harveyi ACP (VhACP), an unconserved Ala75 in WT VhACP, instead of His, destabilizes the structure owing to severe electrostatic repulsion in the absence of divalent cations in the buffer. A75H neutralized this repulsion and induced stable stacking between His75 and Tyr71. Only less than 30 amide protons of VhACP A75H without calcium ions remained after 120 min, whereas most of those VhACP A75Hs in the presence of calcium ions remained even after 6 days, implying that the H/D exchange rates provide important insights into the stability and folding of proteins (Chan et al. 2010). Similarly, via a single mutation of Cys50 to Leu, more amide peaks remained for much longer in the C50L mutant from the residues in the hydrophobic cavity. Compared to the amide protons of WT CaACP, those of C50L CaACP showed slower H/D exchange rates. H/D exchange data along with CD data implied that Cys50 is the key residue in the structural stability and folding of $\mathrm{CaACP}$ and that it contributes to form a wide cavity for BCFA specificity in C. acnes.

Because the membrane composition of CaACP having more BCFAs is different from the membrane composition of most other bacteria, we examined the substrate specificity using CSPs in the HSQC spectra upon conversion from the apo to the linear acyl and branched acyl forms. CSPs for the residues inside of the hydrophobic cavities upon conversion from the apo to the acylated forms clearly indicated that $\mathrm{CaACP}$ can accommodate both linear butyryl and branched isobutyryl chains as substrates. Holo-CaACP showed large CSPs, mainly around the 4'-PP group attachment site, whereas linear butyryl as well as branched isobutyryl CaACP showed large CSPs inside the hydrophobic pockets that were positioned in loop III and helix IV as well as in helix I. The x-ray structure of EcACP revealed that Ile62 and Thr63 form hydrophobic interactions as well as van der Waals interactions with linear acyl groups (Roujeinikova et al. 2007). In this study, Lys67 and Ile68 in CaACP, which correspond to Ile62 and Thr63 in EcACP, respectively, showed large CSPs upon conversion from the apo to the butyryl and isobutyryl forms, confirming that $\mathrm{CaACP}$ has substrate specificity for the branched isobutyryl group. Overall, we can suggest that the unique Cys50 in CaACP is critical factor for the low thermal stability of CaACP but makes enough space in the hydrophobic cavity for substrate specificity. In the future, we will determine the tertiary structure of CaACP; based on the structure, we will investigate the substrate specificity of CaACP and its interaction with other FAS enzymes in detail. We need to 
investigate more detail about the substrate specificity using BCFAs with various branches and lengths using chemical shift perturbations in our future study. We also need to perform conformation-sensitive urea polyacrylamide gel electrophoresis to monitor the reaction of malonyl CaACP and CaKASIII with BCFA-CoA as substrate using E. coli system as a control (Cheon et al. 2019). KASIII of each bacteria have unique cavity structure since each bacteria need various kinds of fatty acids. This implies bacterial KASIII has their own distinctive structural difference for accommodation of CoAs (Qiu et al. 1999). We think high specificity for BCFAs may help to design rationally new antibiotics with specific activity against $C$. acnes.

\section{Conclusions}

In conclusion, in CaACP, Cys50 is the critical residue controlling the thermal and structural stability of CaACP. Because Cys is smaller and more polar than other hydrophobic residues, Cys50 may weaken the stable hydrophobic packing of the cavity, resulting in low structural stability of CaACP. It also induces a loose and wide hydrophobic pocket, which would accept a bulkier branched acyl chain as a substrate. Further studies on the tertiary structures and dynamics of $\mathrm{CaACP}$ and its acylated form will help us better understand the metabolic mechanisms of CaACP. This study may provide insights into understanding the substrate specificity of $C$. acnes and the necessary adaptation mechanisms of $C$. acnes under anaerobic environments. exchanged using $25 \mathrm{mM}$ Mes and $5 \mathrm{mM} \mathrm{CaCl}_{2}$ buffer. CD spectra were measured from 190 to $260 \mathrm{~nm}$ at $0.1-\mathrm{nm}$ intervals and a $100-\mathrm{nm} / \mathrm{min}$ scanning speed. To determine the Tm of CaACP, the mean residue ellipticities $(\theta$, in $\operatorname{deg} \mathrm{cm}^{2} \mathrm{dmole}^{-1}$ ) were derived from the data from three scans obtained between 20 and $75{ }^{\circ} \mathrm{C}$ at $222 \mathrm{~nm}$. Molar ellipticities were plotted with Spectra Manage Version2: Thermal Denaturation Multi Analysis (Jasco, Tokyo, Japan).

\section{Backbone assignment}

To assign the resonances of the backbone atoms and alpha protons of $\mathrm{CaACP}$, we performed $\mathrm{HNCO}$, $\mathrm{HNCACB}, \mathrm{CBCA}(\mathrm{CO}) \mathrm{NH}, 2 \mathrm{D}{ }^{1} \mathrm{H}-{ }^{1} \mathrm{H}$ nuclear Overhauser effect spectroscopy (NOESY), and total correlation spectroscopy (TOCSY) experiments using Bruker Avance $900 \mathrm{MHz}$ spectrometers at KBSI (Ochang, Korea). All NMR spectra were processed with NMRPipe (Delaglio et al. 1995) and analyzed using NMRFAMSparky (Lee et al. 2015).

\section{NMR experiments for CSP measurement and H/D exchange experiments}

To investigate the substrate specificity of CaACP, CSP was measured for $\mathrm{CaACP}$ in ${ }^{1} \mathrm{H}-{ }^{15} \mathrm{~N}$ HSQC spectra using Bruker Avance $700 \mathrm{MHz}$ spectrometers at KBSI (Ochang, Korea) at $25{ }^{\circ} \mathrm{C}$. The NMR samples of CaACP were prepared as described previously (Choi et al. 2021; Lee et al. 2020). The CSPs of apo CaACP and acylated CaACPs were calculated with the following formula:

$$
\Delta \delta=\left(0.5\left(\Delta \delta(1 \mathrm{H})^{2}+\left(\alpha \Delta \delta(15 \mathrm{~N})^{2}\right)\right)\right)^{1 / 2}(\alpha=0.2 \text { for most residues, and } \alpha=0.14 \text { for glycine })
$$

\section{Methods}

\section{Cloning, expression, and purification of $\mathrm{CaACP}$}

The acp $P$ gene was extracted from $C$. acnes genomic DNA and cloned into the pET-21a vector (Novagen, Madison, WI, USA), and the recombinant plasmids were transformed into E. coli BL21(DE3). CaACP was expressed and purified as described previously (Choi et al. 2021; Lee et al. 2020). The acylated ACPs were produced by enzymatic acylation of apo ACPs using holoACP synthase from E. coli and were separated from apo-ACP using a RESOURCE Q column (GE Healthcare, Uppsala, Sweden).

\section{Circular dichroism experiments}

To predict the secondary structure and thermal stability, $\mathrm{CD}$ experiments of $\mathrm{CaACP}$ and its mutants were conducted using a J1500 spectropolarimeter (Jasco, Tokyo, Japan) at the Korea Basic Science Institute (KBSI) (Ochang, Korea). The purified protein samples were totally
To investigate protein folding, we performed H/D exchange experiments as described previously (Lee et al. 2020). Immediately after the addition of $100 \% \mathrm{D}_{2} \mathrm{O}$ to the lyophilized protein sample, HSQC data were acquired every $10 \mathrm{~min}$ for $250 \mathrm{~min}$ for $\mathrm{CaACP}$ and the C50L mutant.

\section{Abbreviations \\ BCFA: Branched-chain fatty acids; CaACP: Cutibacterium acnes acyl carrier protein; Tm: Melting temperature; H/D: Hydrogen/deuterium; WT: Wild- type; HSQC: Heteronuclear single quantum coherence; CSP: Chemical shift perturbation; SFA: Saturated fatty acid; UFA: Unsaturated fatty acid; KASIII: $\beta$-Ketoacyl acyl carrier protein synthase III; EcKASIII: E. coli KASIII; MtKASIII: Mycobacterium tuberculosis KASIII; 4'-PP: 4'-Phosphopantetheine prosthetic; CD: Circular dichroism; CSI: Chemical shift index; SaACP: Staphylococcus aureus ACP; AbACP: Acinetobacter baumannii ACP; EfACP: Enterococcus faecalis ACP; TmACP: Thermotoga maritima ACP; VhACP: Vibrio harveyi ACP.}

\section{Acknowledgements}

We would like to thank the Korea Basic Science Institute (KBSI) for technical assistance with the $700 \mathrm{MHz}$ and $900 \mathrm{MHz}$ NMR experiments under KBSI program (Project No. C140440). 


\section{Authors' contributions}

YK contributed to conceptualization, writing —original draft preparation; AJ contributed to data analysis, visualization, writing — original draft preparation; EH contiributed to data analysis; AJ and YK contributed to writing - review and editing; YK contributed to supervision and funding acquisition; All authors read and approved the final manuscript.

\section{Funding}

This work was supported by the National Research Foundation of Korea (NRF) grant funded by the Korea government (MSIT) (No: 2020R1A2C2005338).

\section{Availability of data and materials}

The data presented in this study are available on request from the corresponding author.

\section{Declarations}

\section{Competing interests}

The authors declare that they have no competing interests.

\section{Author details}

${ }^{1}$ Department of Bioscience and Biotechnology, Konkuk University, Seoul 05029, Republic of Korea. ${ }^{2}$ Bio-Chemical Analysis Team, Korea Basic Science Institute, Cheongju, Chungbuk 28119, Republic of Korea.

Received: 16 November 2021 Accepted: 4 December 2021 Published online: 04 January 2022

\section{References}

Andrec M, Hill RB, Prestegard JH. Amide exchange rates in Escherichia coli acyl carrier protein: correlation with protein structure and dynamics. Protein Sci. 1995;4(5):983-93. https://doi.org/10.1002/pro.5560040518.

Aubin GG, Baud'huin M, Lavigne JP, Brion R, Gouin F, Lepelletier D, Jacqueline C, Heymann D, Asehnoune K, Corvec S. Interaction of Cutibacterium (formerly Propionibacterium) acnes with bone cells: a step toward understanding bone and joint infection development. Sci Rep. 2017;7:42918. https://doi. org/10.1038/srep42918.

Bogardt RA Jr, Jones BN, Dwulet FE, Garner WH, Lehman LD, Gurd FR. Evolution of the amino acid substitution in the mammalian myoglobin gene. J Mol Evol. 1980;15(3):197-218. https://doi.org/10.1007/BF01732948.

Boisrenoult P. Cutibacterium acnes prosthetic joint infection: diagnosis and treatment. Orthop Traumatol Surg Res. 2018;104(1S):S19-24. https://doi.org/10. 1016/j.otsr.2017.05.030.

Chan DI, Stockner T, Tieleman DP, Vogel HJ. Molecular dynamics simulations of the Apo-, Holo-, and acyl-forms of Escherichia coli acyl carrier protein. J Biol Chem. 2008;283(48):33620-9. https://doi.org/10.1074/jbc.M805323200.

Chan DI, Chu BC, Lau CK, Hunter HN, Byers DM, Vogel HJ. NMR solution structure and biophysical characterization of Vibrio harveyi acyl carrier protein $\mathrm{A} 75 \mathrm{H}$ : effects of divalent metal ions. J Biol Chem. 2010;285(40):30558-66. https:// doi.org/10.1074/jbc.M110.128298.

Cheon D, Lee WC, Lee Y, Lee JY, Kim Y. Structural basis of branched-chain fatty acid synthesis by Propionibacterium acnes beta-ketoacyl acyl Carrier protein synthase. Biochem Biophys Res Commun. 2019;509(1):322-8. https://doi. org/10.1016/j.bbrc.2018.12.134.

Choi S, Park J, Yeon J, Jang A, Lee WC, Kim Y. Deciphering the binding interactions between Acinetobacter baumannii ACP and beta-ketoacyl ACP synthase III to improve antibiotic targeting using NMR spectroscopy. Int J Mol Sci. 2021. https://doi.org/10.3390/ijms22073317.

Delaglio F, Grzesiek S, Vuister GW, Zhu G, Pfeifer J, Bax A. NMRPipe: a multidimensional spectral processing system based on UNIX pipes. J Biomol NMR. 1995;6(3):277-93. https://doi.org/10.1007/BF00197809.

Duldhardt I, Gaebel J, Chrzanowski L, Nijenhuis I, Hartig C, Schauer F, Heipieper HJ. Adaptation of anaerobically grown Thauera aromatica, Geobacter sulfurreducens and Desulfococcus multivorans to organic solvents on the level of membrane fatty acid composition. Microb Biotechnol. 2010;3(2):201-9. https://doi.org/10.1111/j.1751-7915.2009.00124x.

Eghbalnia HR, Wang L, Bahrami A, Assadi A, Markley JL. Protein energetic conformational analysis from NMR chemical shifts (PECAN) and its use in determining secondary structural elements. J Biomol NMR. 2005;32(1):7181. https://doi.org/10.1007/s10858-005-5705-1.

Gharamti AA, Kanafani ZA. Cutibacterium (formerly Propionibacterium) acnes infections associated with implantable devices. Expert Rev Anti Infect Ther. 2017;15(12):1083-94. https://doi.org/10.1080/14787210.2017.1404452.

Heath RJ, Rock CO. Inhibition of beta-ketoacyl-acyl carrier protein synthase III (FabH) by acyl-acyl carrier protein in Escherichia coli. J Biol Chem. 1996;271(18):10996-1000. https://doi.org/10.1074/jbc.271.18.10996.

Kaspar HF. Nitrite reduction to nitrous oxide by propionibacteria: Detoxication mechanism. Arch Microbiol. 1982;133(2):126-30. https://doi.org/10.1007/ bf00413525.

Krishna MM, Hoang L, Lin Y, Englander SW. Hydrogen exchange methods to study protein folding. Methods. 2004;34(1):51-64. https://doi.org/10.1016/j. ymeth.2004.03.005

Lai CY, Cronan JE. Beta-ketoacyl-acyl carrier protein synthase III (FabH) is essential for bacterial fatty acid synthesis. J Biol Chem. 2003;278(51):51494-503. https://doi.org/10.1074/jbc.M308638200.

Lee W, Tonelli M, Markley JL. NMRFAM-SPARKY: enhanced software for biomolecular NMR spectroscopy. Bioinformatics. 2015;31(8):1325-7. https://doi. org/10.1093/bioinformatics/btu830.

Lee Y, Jang A, Jeong MC, Park N, Park J, Lee WC, Cheong C, Kim Y. Structural characterization of an ACP from Thermotoga maritima: insights into hyperthermal adaptation. Int J Mol Sci. 2020. https://doi.org/10.3390/ijms21072600.

Moss CW, Dowell VR Jr, Farshtchi D, Raines L, Cherry WB. Cultural characteristics and fatty acid composition of propionibacteria. J Bacteriol. 1969;97(2):56170. https://doi.org/10.1128/jb.97.2.561-570.1969.

Niklasson M, Andresen C, Helander S, Roth MG, Zimdahl Kahlin A, Lindqvist Appell M, Martensson LG, Lundstrom P. Robust and convenient analysis of protein thermal and chemical stability. Protein Sci. 2015;24(12):2055-62. https://doi.org/10.1002/pro.2809.

Park YG, Jung MC, Song H, Jeong KW, Bang E, Hwang GS, Kim Y. Novel structural components contribute to the high thermal stability of acyl carrier protein from Enterococcus faecalis. J Biol Chem. 2016;291(4):1692-702. https://doi. org/10.1074/jbc.M115.674408.

Ploskon E, Arthur CJ, Kanari AL, Wattana-amorn P, Williams C, Crosby J, Simpson TJ, Willis CL, Crump MP. Recognition of intermediate functionality by acyl carrier protein over a complete cycle of fatty acid biosynthesis. Chem Biol. 2010;17(7):776-85. https://doi.org/10.1016/j.chembiol.2010.05.024.

Qiu X, Janson CA, Konstantinidis AK, Nwagwu S, Silverman C, Smith WW, Khandekar S, Lonsdale J, Abdel-Meguid SS. Crystal structure of beta-ketoacyl-acyl carrier protein synthase III. A key condensing enzyme in bacterial fatty acid biosynthesis. J Biol Chem. 1999;274(51):36465-71. https://doi.org/ 10.1074/jbc.274.51.36465.

Rock CO, Cronan JE. Escherichia coli as a model for the regulation of dissociable (type II) fatty acid biosynthesis. Biochim Biophys Acta. 1996;1302(1):1-16. https://doi.org/10.1016/0005-2760(96)00056-2.

Roujeinikova A, Simon WJ, Gilroy J, Rice DW, Rafferty JB, Slabas AR. Structural studies of fatty acyl-(acyl carrier protein) thioesters reveal a hydrophobic binding cavity that can expand to fit longer substrates. J Mol Biol. 2007;365(1):13545. https://doi.org/10.1016/j.jmb.2006.09.049.

Scarsdale JN, Kazanina G, He X, Reynolds KA, Wright HT. Crystal structure of the Mycobacterium tuberculosis beta-ketoacyl-acyl carrier protein synthase III. J Biol Chem. 2001:276(23):20516-22. https://doi.org/10.1074/jbc.M010762200.

Shaw MK, Ingraham JL. Fatty acid composition of Escherichia coli as a possible controlling factor of the minimal growth temperature. J Bacteriol. 1965;90(1):141-6. https://doi.org/10.1128/jb.90.1.141-146.1965.

Wishart DS, Sykes BD. The 13C chemical-shift index: a simple method for the identification of protein secondary structure using $13 \mathrm{C}$ chemical-shift data. J Biomol NMR. 1994;4(2):171-80. https://doi.org/10.1007/BF00175245.

Wishart DS, Sykes BD, Richards FM. The chemical shift index: a fast and simple method for the assignment of protein secondary structure through NMR spectroscopy. Biochemistry. 1992;31(6):1647-51. https://doi.org/10.1021/ bi00121a010.

Zhang YM, Rock CO. Membrane lipid homeostasis in bacteria. Nat Rev Microbiol. 2008;6(3):222-33. https://doi.org/10.1038/nrmicro1839.

\section{Publisher's Note}

Springer Nature remains neutral with regard to jurisdictional claims in published maps and institutional affiliations. 